\title{
Insertos ultrassônicos na desobstrução de canais com pinos de fibra de vidro: estudo
}

\section{in vitro}

\author{
Ultrasonic inserts in channel cleaning with fiber posts: in vitro study \\ Insertos ultrasónicos en la limpieza de canales con pins de fibra de vidrio: estudio in vitro
}

Recebido: 21/10/2020 | Revisado: 27/10/2020 |Aceito: 11/02/2021 | Publicado: 19/02/2021

\author{
Esdras Gabriel Alves-Silva \\ ORCID: https://orcid.org/0000-0002-2309-1115 \\ Universidade Estadual de Campinas, Brasil \\ E-mail: esdras0702@yahoo.com.br \\ Joseane Beatriz Gurgel de Medeiros \\ ORCID: https://orcid.org/0000-0001-7335-9477 \\ Universidade Federal do Rio Grande do Norte, Brasil \\ E-mail: joseanegurgel@hotmail.com \\ Warlenya Duarte de Medeiros \\ ORCID: https://orcid.org/0000-0001-8841-8513 \\ Universidade Potiguar, Brasil \\ E-mail: warlenyaduarte_001@hotmail.com \\ Fábio Roberto Dametto \\ ORCID: https://orcid.org/0000-0001-8108-8523 \\ Universidade Federal do Rio Grande do Norte, Brasil \\ E-mail: dametto71@hotmail.com \\ Rodrigo Arruda-Vasconcelos \\ ORCID: https://orcid.org/0000-0002-0968-0212 \\ Universidade Estadual de Campinas, Brasil \\ E-mail: vasconcelosra@yahoo.com.br \\ Lidiane Mendes Louzada \\ ORCID: https://orcid.org/0000-0002-3480-8433 \\ Universidade Estadual de Campinas, Brasil \\ E-mail: lidiane.mlouzada@gmail.com \\ Brenda Paula Figueiredo de Almeida Gomes \\ ORCID: https://orcid.org/0000-0002-8449-0646 \\ Universidade Estadual de Campinas, Brasil \\ E-mail: bpfagomes@fop.br \\ Cícero Romão Gadê-Neto \\ ORCID: https://orcid.org/0000-0001-6043-2110 \\ Universidade Federal do Rio Grande do Norte, Brasil \\ E-mail: gadeneto@yahoo.com
}

\begin{abstract}
Resumo
Atualmente a escolha de uma adequada restauração de dentes tratados endodonticamente é guiada em parte pela exigência estética. Os pinos intrarradiculares são utilizados quando o remanescente dental não possui um suporte adequado na retenção. Diante das dificuldades para remoção dos pinos de fibra de vidro. O objetivo deste trabalho, in vitro, foi comparar duas técnicas de desgaste de pino de fibra de vidro para desobstrução de canais radiculares. Avaliou-se o tempo para remoção do pino de fibra de vidro. Foram selecionados 26 dentes unirradiculares padronizados em 15 milímetros de comprimento real de trabalho (CRT). O Preparo químico-mecânico foi realizado com o sistema Protaper universal. Após obturação, foi desobturado $10 \mathrm{~mm}$ do canal, deixando $5 \mathrm{~mm}$ de material remanescente no terço apical. Os pinos foram cimentados de acordo com as orientações do fabricante. Os dentes foram divididos em dois grupos para a remoção do pino: No Grupo 01 (G1), o desgaste de até $7 \mathrm{~mm}$ do pino foi realizado utilizando uma broca esférica multilaminada $\mathrm{n}^{\circ} 1(\mathrm{LN})$, em baixa rotação, associado ao inserto ultrassônico tronco-cônico TRI 01 DA3, sob refrigeração, para o desgaste do remanescente de pino. No Grupo 02 (G2) o desgaste do pino foi feito utilizando o inserto ultrassônico tronco-cônico liso PERIOSUB, sob refrigeração. Foi realizado a cronometragem durante a remoção do pino de fibra de vidro em que G1 obteve média de 290 segundos e o G2 com média de 753 segundos estatisticamente significativo (Test $t$ não pareado $p<0,05$ ). $O$ grupo G1 apresentou menor tempo para remoção do pino de fibra de vidro quando comparado com o G2. A melhor opção para remoção do pino de fibra de vidro parece ser a associação da broca esférica seguido do inserto ultrassônico na remoção de pinos de fibra de vidro.
\end{abstract}

Palavras-chave: Inserto ultrassônico; Pinos de fibra de vidro; Retratamento endodôntico. 


\begin{abstract}
Currently, the choice of an adequate restoration of endodontically treated teeth is guided in part by the aesthetic requirement. Intraradicular pins are used when the dental remnant does not have adequate support for retention. In view of the difficulties in removing the fiberglass pins. The objective of this work, in vitro, was to compare two techniques of fiberglass pin wear to unclog root canals. Materials and Methods: the time to remove the fiberglass pin was evaluated. 26 standard single-toothed teeth were selected in $15 \mathrm{~mm}$ real working length (CRT). The chemicalmechanical preparation was carried out with the universal Protaper system. After filling, $10 \mathrm{~mm}$ of the canal was cleared, leaving $5 \mathrm{~mm}$ of material remaining in the apical third. The pins were cemented according to the manufacturer's guidelines. The teeth were divided into two groups for the removal of the pin: In Group 01 (G1), the wear of up to $7 \mathrm{~mm}$ of the pin was performed using a multilaminated spherical drill No. 1 (LN), in low rotation, associated with the ultrasonic insert - conical TRI 01 DA3, under refrigeration, to wear the remaining pin. In Group 02 (G2) the wear of the pin was made using the PERIOSUB smooth trunk-conical ultrasonic insert, under refrigeration. Timing was performed during the removal of the fiberglass pin, where G1 obtained an average of 290 seconds and G2 with an average of 753 seconds, which was statistically significant (unpaired t test $p<0.05$ ). The G1 group had a shorter time for removing the fiberglass pin when compared to G2. The best option for removing the fiberglass pin seems to be the association of the spherical drill followed by the ultrasonic insert in the removal of fiberglass pins.
\end{abstract}

Keywords: Ultrasonic Insert; Fiberglass pins; Endodontic retreatment.

\title{
Resumen
}

Actualmente, la elección de una restauración adecuada de los dientes tratados con endodoncia se guía en parte por los requisitos estéticos. Los pines intrarradiculares se utilizan cuando el remanente dental no tiene el soporte adecuado para la retención. En vista de las dificultades para retirar los pasadores de fibra de vidrio. El objetivo de este trabajo, in vitro, fue comparar dos técnicas de desgaste de pasadores de fibra de vidrio para desbloquear conductos radiculares. Se evaluó el tiempo para quitar el pasador de fibra de vidrio. Se seleccionaron 26 dientes unirradiculares estándar en $15 \mathrm{~mm}$ de longitud de trabajo real (TRC). La preparación químico-mecánica se realizó con el sistema Protaper universal. Después del llenado, se despejaron $10 \mathrm{~mm}$ del canal, quedando $5 \mathrm{~mm}$ de material en el tercio apical. Los pasadores se cementaron de acuerdo con las pautas del fabricante. Los dientes se dividieron en dos grupos para la extracción del pasador: En el Grupo $01(\mathrm{G} 1)$, se realizó el desgaste de hasta $7 \mathrm{~mm}$ del pasador mediante una fresa esférica multilaminada núm. - TRI 01 DA3 cónico, refrigerado, para el desgaste del perno restante. En el Grupo 02 (G2) el desgaste del perno se realizó mediante el inserto ultrasónico tronco-cónico liso PERIOSUB, bajo refrigeración. El cronometraje se realizó durante la extracción de la clavija de fibra de vidrio, con G1 promediando 290 segundos y G2 promediando 753 segundos, lo cual fue estadísticamente significativo (prueba t no apareada $p<$ 0.05). El grupo G1 tuvo menos tiempo para quitar el pasador de fibra de vidrio en comparación con el G2. La mejor opción para quitar el pasador de fibra de vidrio parece ser la asociación del taladro esférico seguido del inserto ultrasónico en la extracción de los pasadores de fibra de vidrio.

Palabras clave: Inserto ultrasónico; Pasadores de fibra de vidrio; Retratamiento endodóntico.

\section{Introdução}

Os casos de dentes tratados endodonticamente com uma considerável perda de estrutura coronária, ao qual o remanescente dental já não pode fornecer suporte adequado, necessitam de pinos radiculares. Nas condições de grande destruição coronária, o agente de fixação é insuficiente para garantir retenção e o artefato empregado para assegurar que o núcleo não se desloque chama-se pino radicular (Kalkan et al,2006). O alojamento deste situa-se no canal radicular e quando são rígidos, como o metálico fundido com ligas de níquel-cromo, ao serem colocados em dentes tratados endodonticamente conflitam com a constituição biomecânica do dente (Balbosh et al,2006).

Existe uma grande diversidade de pinos disponíveis como os rosqueáveis em dentina, pinos pré-fabricados e metálicos fundidos. Até recentemente, os pinos metálicos fundidos eram muito populares devido a algumas vantagens como uma boa rigidez, baixo custo, técnica simplificada, o fato de não necessitar de cimentos especiais na sua fixação, larga experiência clínica e uma excelente radiopacidade. Porém, suas desvantagens como ausência de estética, a possibilidade de sofrer corrosão, alto módulo de elasticidade e o fato de não serem adesivos (Balbosh,2006; Artopoulou et al,2006; Garrido,2007) aumentam a possibilidade de insucesso das reabilitações dentárias. Os pinos rosqueáveis em dentina apresenta um grande risco de invadir o espaço biológico através de perfuração cervical, ou mesmo do assoalho da câmara pulpar (Braga et al,2005). Com isso, tem-se 
procurado por materiais com características similares ao módulo de elasticidade da dentina com o objetivo de aumentar a longevidade das restaurações indiretas (Kalkan et al,2006; Braga et al,2005).

Há alguns anos, foram introduzidos no mercado diversos sistemas de pinos reforçados por fibras que, em geral, são compostos por fibras de quartzo, sílica ou vidro embebidas em matriz de resina epóxica ou metacrilato. Dentre eles, o pino de fibra de vidro possui como vantagens módulo de elasticidade semelhante à dentina radicular (Artopoulou et al,2006), não ser corrosivo, ter biocompatibilidade (Goodacre,2010), ser estético e translúcido, recomendados principalmente para dentes anteriores. Dentes restaurados com pino de fibra de vidro apresentam menor frequência de fracassos do que dentes restaurados com pinos e núcleos metálicos após 4 anos de uso clínico (Goodacre,2010).

O pino de fibra de vidro é cimentado com o uso de um cimento resinoso, que, além de preservar e reforçar o remanescente dental permite uma melhor distribuição de tensões, além de melhorar a retenção e a estética (Muniz,2005). Contudo, a fase de cimentação desses pinos pode ser considerada o ponto mais vulnerável do procedimento restaurador indireto, por isso têm sido cada vez mais aprimorado em busca da máxima eficiência(Rocha,2016), causando assim, dificuldade na remoção do pino caso necessário, tornando-o um problema a mais em casos de retratamento endodôntico (Rocha,2016).

A presença de um pino de fibra de vidro em um dente tratado endodonticamente que será submetido ao retratamento endodôntico gera uma dificuldade considerável à execução da técnica, por fatores, como: material, forma e comprimento do pino, tipo de cimento utilizado na cimentação, a inter-relação do pino com as paredes do canal, a acessibilidade, bem como a habilidade dos recursos profissionais e técnicos disponíveis para a remoção (Soares et al,2009).

Com o propósito de facilitar a remoção do pino radicular, várias técnicas de remoção surgiram, e com elas, o uso de inserto ultrassônico, considerado mais fácil e seguro, por apresentar vantagens como um mínimo desgaste de estrutura dentária, menor tempo de trabalho, menor risco de perfuração de raiz e de fraturas, e fácil manuseio do ultrassom (Rijk,2000). Portanto, sabendo da dificuldade que o pino de fibra confere ao retratamento endodôntico (Lindemann et al,2005), e que as técnicas de remoção do pino de fibra de vidro utilizando insertos ultrassônicos são mais eficazes, o objetivo deste trabalho, in vitro, foi comparar duas técnicas de desgaste de pino de fibra de vidro para desobstrução de canais radiculares obturados endodonticamente, utilizando a cronometragem para avaliar o tempo de trabalho; e o exame radiográfico antes, durante e após a desobstrução dos pinos das amostras para avaliar o desgaste interno.

\section{Metodologia}

\section{Aspectos éticos}

Este estudo experimental in vitro, laboratorial e randomizado (Pereira et al,2018) e seguindo os preceitos estabelecidos pela resolução 196/96 do Ministério da saúde, este estudo foi devidamente registrado na base de registo de pesquisa envolvendo seres humanos (Plataforma Brasil) e submetido à apreciação e aprovação do comitê de ética e pesquisa da Universidade Potiguar- UnP/Natal/RN, com o número do CAAE 43207915.2.0000.5296.

\section{Tempo}

O tempo para desobstrução dos canais através do desgaste dos pinos foi avaliado através da cronometragem de cada técnica avaliada.

\section{Seleção das amostras}

Foram selecionados 26 dentes unirradiculares humanos, extraídos, superiores e inferiores, rizogênese completa, sem curvaturas acentuadas, sem trincas, sem tratamento endodôntico prévio. 


\section{Esterilização das amostras}

Foram armazenados em timol a $0,1 \%, \mathrm{pH}$ neutro, com finalidade de hidratação e evitar o crescimento de fungos. Os dentes foram autoclavados em um recipiente semi-aberto com água destilada. Após autoclavados, foram limpos com auxílio de curetas periodontais.

\section{Tomadas radiográficas}

Os dentes foram posicionados em filmes oclusais e foram feitas tomadas nas incidências M/D (mésio/distal) e V/L (vestíbulo/lingual) para análise do comprimento, curvaturas e diâmetro dos canais. A partir das análises radiográficas dentes com discrepâncias de tamanho, curvatura e diâmetro foram descartados da amostra.

\section{Padronização das amostras}

As coroas dos dentes foram seccionadas com o auxílio de disco de aço, de forma que o remanescente radicular fosse padronizado em 16 milímetros $(\mathrm{mm})$ para todos os dentes. Para a realização do tratamento endodôntico, uma lima tipo K\#10 (Dentsply Maillefer, Ballaigues, Switzerland) foi introduzida no interior do canal com movimentos de cateterismo, até ser visualizada no forame apical, obtendo-se o comprimento real do canal. E seguida à lima foi recuada $\mathrm{em} 1 \mathrm{~mm}$, estabelecendose o comprimento real de trabalho (CRT), $1 \mathrm{~mm}$ aquém do forame apical $(15 \mathrm{~mm})$.

\section{Tratamento Endodôntico}

A execução do Preparo químico-mecânico foi realizado por um único operador especialista em endodontista experiência clínica utilizado o motor elétrico X-Smart (Dentsply Maillefer, Ballaigues, Switzerland), em uma rotação constante, velocidade de 250 rotações por minuto (rpm) e torque de 3 Newton por centímetro (n), com o sistema de limas Protaper de níquel-titânio (Dentsply Maillefer, Ballaigues, Switzerland). Técnica de preparo: Pré-alargamento do terço cervical e médio com a lima SX (aproximadamente $10 \mathrm{~mm}$ ), sequenciando com as limas S1, S2, F1, F2 e F3 no CRT (em 15 milímetros). A cada troca de lima foi utilizado uma seringa descartável com agulha (BD 0,55 x 20-24G 3/4), para irrigação do canal radicular com $2 \mathrm{~mL}$ de solução irrigadora de hipoclorito de sódio 2,5\%. Para a remoção da smearlayer o canal foi preenchido com solução de EDTA Tri-sódico $17 \%$ por 3 minutos, sendo o primeiro minuto agitado com lima $\mathrm{K}$ n$^{\circ} 10$ no CRT. Logo após, foi feito irrigação com $2 \mathrm{~mL}$ de Hipoclorito de Sódio 2,5\% e lavagem final com $2 \mathrm{~mL}$ de Soro Fisiológico. Ao final, os canais foram secos com cones de papel no CRT.

\section{Obturação dos canais radiculares}

Foi realizada a obturação dos canais utilizando o cimento obturador Sealer 26 (Dentsply® Vila Gertrudes, São Paulo, SP, Brasil). Este foi preparado de acordo com as orientações do fabricante. Com o auxílio de uma lima K\#30 (Dentsply Maillefer, Ballaigues, Switzerland) o cimento Sealer 26 (Dentsply Maillefer, Ballaigues, Switzerland) foi levado ao interior do canal radicular. Em seguida o cone de guta-percha F3 (Dentsply®), previamente carregado com o cimento Sealer 26 (Dentsply Maillefer, Ballaigues, Switzerland), pela técnica do cone modelado apical, complementada com a condensação lateral. Foi deixado um espaço de $2 \mathrm{~mm}$ na embocadura do canal, para colocar um plugue de material selador temporário Coltosol (Vigodent, Coltene, Rio de Janeiro, RJ). As amostras foram acondicionadas em frascos plásticos, envoltas em gaze com 100\% de umidade e armazenadas em estufa laboratorial a $37^{\circ} \mathrm{C}$ por 72 horas, obedecendo-se o tempo de presa do cimento obturador. 


\section{Preparo do espaço protético e cimentação dos pinos}

Após decorrido o tempo de presa do cimento endodôntico, com auxílio da Broca esférica diamandata 1011 foi retirado o plugue de material selador temporário Coltosol e com a broca Largo ${ }^{\circ} 4$ desobturou-se $10 \mathrm{~mm}$ do canal radicular, deixando 5 $\mathrm{mm}$ de material remanescente no terço apical, seguido do preparo do conduto, que foi realizado com a broca Exacto ${ }^{\circ} 2$ ( $3 \mathrm{M}$ Dental Product, St Paul, USA), referente aos pinos de fibra de vidro utilizados. Os pinos de fibra de vidro utilizados foram EXACTO n² Angelus (3M Dental Product, St Paul, USA), no qual foram limpos com álcool 70\%, seguido da aplicação do silano $(F G M \circledast)$ e então, deixou-se secar por $1 \mathrm{~min}$. Em seguida, foi realizado o condicionamento ácido do conduto com ácido fosfórico $37 \%$ por $15 \mathrm{~s}$, seguido de lavagem e secagem com cones de papel absorvente. Com microbrush, foi aplicado o sistema adesivo (3M Dental Product, St Paul, USA), retirado o excesso com pontas de papel absorvente, seco com leve jato de ar por 10s e fotoativado por 20s. As pastas (base e catalisadora) do cimento Allcem (FGM Vila Centenário, São Paulo, SP, Brasil) foram manipuladas em partes iguais e aplicadas no conduto com auxílio de uma lima K\#20 até o preenchimento do conduto, e no pino. $\mathrm{O}$ pino foi inserido no conduto e fotopolimerizado por 40s. As amostras foram levadas à estufa microbiológica por 48 horas.

\section{Desgaste do pino: Os dentes foram divididos em dois grupos.}

Grupo 01 (G1):

No Grupo 01 (G1) o desgaste do pino foi feito utilizando uma broca esférica multilaminada ${ }^{\mathrm{a}} 1$ (LN) (Figura1), em baixa rotação, com o desgaste dos $0,3 \mathrm{~mm}$ restantes do pino utilizando o aparelho ProfiNeo (DabiAtlante®), em potência máxima, com o inserto ultrassônico tronco-cônico TRI 01 DA3 (Trinks, São Paulo, São Paulo, Brasil), sob refrigeração, como mostra na Figura 1.

Figura 1. A. Broca esférica multilaminada na 1. B. Inserto ultrassônico tronco-cônico TRI 01 DA3

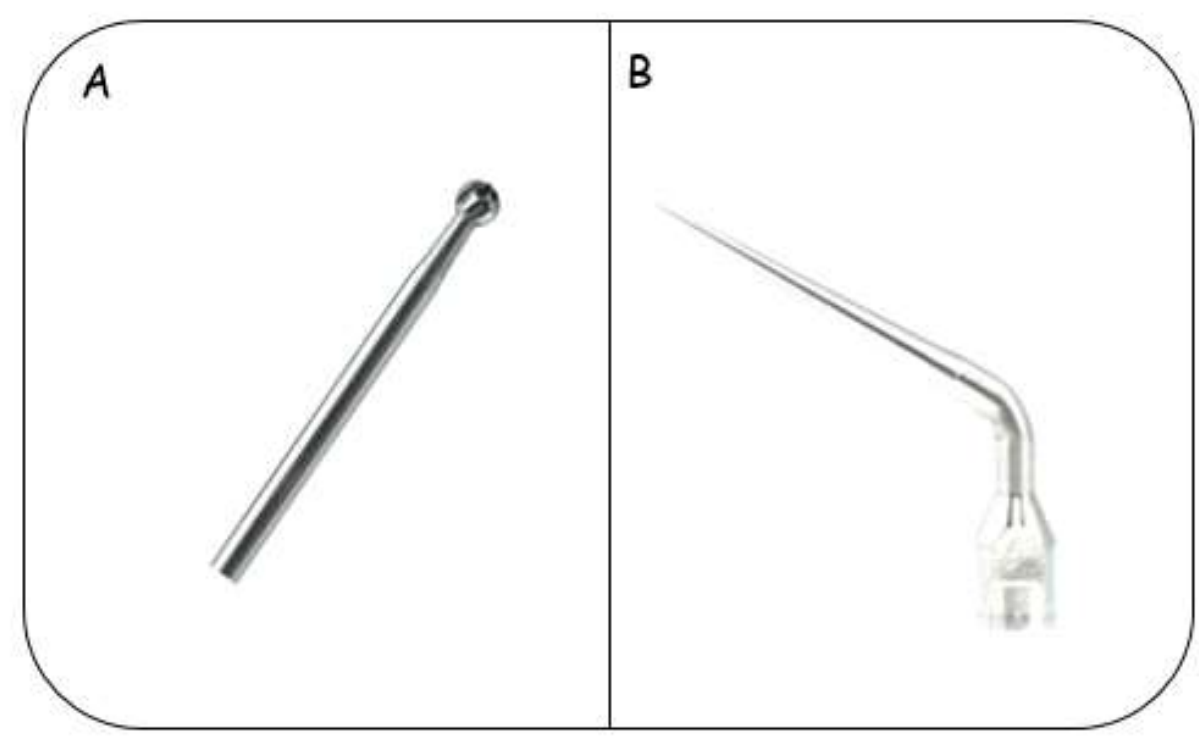

Fonte: Autores. 
Grupo $02(\mathrm{G} 2)$ :

No Grupo 02 (G2) o desgaste do pino foi feito utilizando o aparelho ProfiNeo (DabiAtlante®), em potência máxima, com o inserto ultrassônico tronco- cônico liso PERIO SUB (DabiAtlante ${ }^{\circledR}$ ), sob refrigeração, como mostra na figura 2.

Figura 2. Inserto ultrassônico tronco-cônico liso Perio Sub.

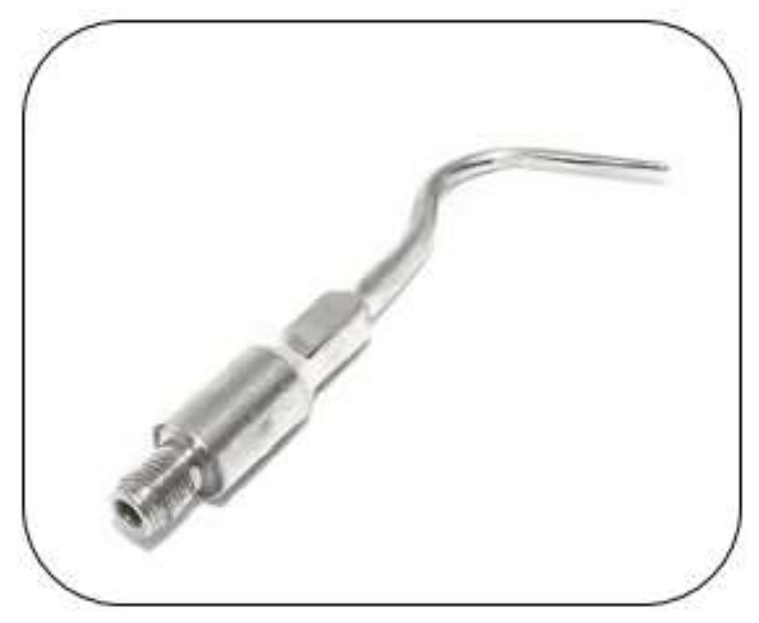

Fonte: Autores.

\section{Análise das amostras}

Em todos os procedimentos de desobstrução foi utilizado microscópio clínico (D.F Vasconcelos®) e a cronometragem foi feita no início da remoção do pino de fibra de vidro até a visualização da guta-percha de todas as amostras.

\section{Resultados e Discussão}

Tempo de Desobstrução: o Grupo G1 teve média de 290 segundos e o grupo G2 com média de 753 segundos para remover todo material do canal radicular, como mostra a Tabela 1

Tabela 1. Média e desvio padrão do tempo em segundos de desobturação.

\begin{tabular}{lcc}
\hline Variáveis & Média & Desvio Padrão \\
\hline G1 & $290^{\mathrm{b}}$ & 95 \\
G2 & $753^{\text {a }}$ & 84 \\
\hline
\end{tabular}

Brocas Multilaminadas n*1(G1); Insertos Ultrassônicos PerioSub(G2). Letras diferentes indicam diferença estatística significante test t não pareado $(\mathrm{p}<0,0001)$. Fonte: Autores.

Em decorrência da grande dificuldade que a desobstrução do pino de fibra de vidro confere ao retratamento endodôntico, o objetivo deste trabalho foi proporcionar ao cirurgião dentista maior discernimento de cada técnica operatória, e alguns de seus resultados de interesse clínico. Para isso, é necessário conhecer e avaliar técnicas indicadas à resolutividade do caso e que estejam ao alcance do cirurgião dentista (Goodacre, 2010; Rocha, 2016).

O G1 foi o grupo que mostrou os melhores resultados com relação ao tempo de desobstrução dos canais, com a média de 290 segundos, e o G2, com média de 753 segundos, o que confirma os resultados do estudo de Coelho, (2012), em que apresentou o menor tempo de trabalho no grupo que foi utilizado o inserto ultrassônico diamantado, com uma média de 120 segundos. Já Lindemann et al, (2005), mostraram em seu estudo que o grupo em que o ultrassom foi utilizado na desobstrução 
teve um tempo adicional de 10 min. O fato da broca carbide ter a ação de corte e estar associado ao inserto diamantado, que tem ação de desgaste, além da forma tronco-cônica do inserto, que facilita a penetração no pino (Gesi et al,2003), podem justificar os resultados.

Segundo Fracassi et al, (2010), a remoção de pinos é um exercício exaustivo e complicado, onde vários fatores podem influenciar, como a acessibilidade do pino, sua relação com as paredes do conduto, o cimento usado, e entre outros, a habilidade do profissional. Sabendo disso, em relação às dificuldades encontradas na técnica, pode-se perceber, além destas já citadas, que a translucidez do pino dificulta a definição dos limites de onde se pode atuar ou não, podendo também ter influenciado na quantidade de desgaste excessivo.

Lindemann et al, (2005), avaliaram e compararam a eficácia da remoção de três tipos de pinos de fibra de vidro e um tipo de pino metálico. Após avaliação, os resultados sugeriram que os insertos ultrassônicos são mais eficazes na melhoria da qualidade da remoção. No entanto, neste estudo observou-se que a broca diamantada mostrou-se com um tempo menor em relação ao inserto ultrassônico na remoção do pino de fibra de vidro.

A média de tempo de remoção dos pinos encontrados nesta pesquisa para o grupo que utilizou o inserto ultrassônico está de acordo com os achados de Fracassi et al, (2010), aplicaram o ultrassom em pino metálico com o intuito de trincar e remover o cimento resinoso e o ultrassom foi aplicado em pino de fibra de vidro com o intuito de desgastar o pino até a sua porção mais profunda, sendo também cimentado com cimento resinoso dual.

Em sua pesquisa Rocha (2016), avaliou a eficácia e eficiência da remoção de pino de fibra de vidro, cimentados com cimento resinoso, em dentes decíduos bovinos tratados endodonticamente. Utilizou três instrumentos para a remoção dos pinos, broca diamantada esférica, broca específica de preparo do conduto para pinos White post 2.0 FGM e inserto ultrassônico cônico longo. Os resultados encontrados por Rocha (2016), corroboram com os resultados desta pesquisa que encontrou que o uso da broca esférica removeu o pino de forma mais rápida, porém sem eficácia; e que o inserto ultrassônico promoveu o maior tempo clínico de remoção de todos os instrumentos de remoção pesquisados.

A utilização do microscópio operatório como auxílio nos procedimentos de desobstrução dos pinos de fibra de vidro proporcionou uma melhor visualização dos detalhes do conduto que não podem ser observados a olho nu, tanto pela iluminação como pelo aumento da magnitude, o que trouxe mais segurança ao procedimento, diminuindo assim, a possibilidade de mais desvios (Benassi et al, 2008; Kim et al, 2016).

A realização das radiografias transoperatórias foi outro importante auxílio no controle da trajetória de desobstrução, onde foi possível comparar com as radiografias prévias e observar quais pontos estavam mais sujeitos ao desvio ou não, conferindo a possibilidade de correção ou mesmo de prosseguir com o trajeto (Kim et al,2016).

De acordo com Oliveira et al,2012 o cimento resinoso dual apresenta propriedades de resistência adesiva à dentina, resistência adesiva ao esmalte, resistência ao desgaste, entre outras, justificando o uso do cimento resinoso Allcem (FGM), onde este passa por um processo de polimerização e reação química, no qual, conclui sua presa em regiões profundas que a luz não consegue alcançar.

Segundo Lopes e Siqueira,2010 o pino deve ter um comprimento ideal que seja 2/3 do comprimento do remanescente dental e que o selamento apical seja de 3 a $5 \mathrm{~mm}$ de obturação radicular. Então, com a secção das amostras do presente estudo em $16 \mathrm{~mm}$ e definindo o comprimento real de trabalho em $15 \mathrm{~mm}$, foi seguido o princípio biomecânico quando foi deixado 5 $\mathrm{mm}$ de selamento apical e $10 \mathrm{~mm}$ de pino de fibra de vidro cimentados no conduto, corroborando com os resultados de Gonçalves et al, (2012).

A escolha do cimento endodôntico Sealer 26 nessa pesquisa deu-se pelas suas boas propriedades biomecânicas relatadas nos estudos de Silva et al, (2015), onde concluíram que os cimentos a base de resina epóxica (Sealer 26 e H- plus) 
apresentam menor infiltração apical quando comparado a outros cimentos endodônticos como N-Ricket e RSA. Além disso também relataram em seu estudo sua eficácia no selamento apical (Silva et al,2012).

\section{Considerações Finais}

De acordo com a metodologia aplicada e limitações deste estudo, pode-se concluir que:

- O grupo G1 apresentou menor tempo para remoção do pino de fibra de vidro quando comparado com o G2; A melhor opção para remoção do pino de fibra de vidro parece ser a associação da broca esférica seguido do inserto ultrassônico na remoção de pinos de fibra de vidro.

\section{Agradecimentos}

Este trabalho foi financiado pela Fundação de Amparo à Pesquisa do Estado de São Paulo (FAPESP) (número de financiamento: 2015 / 23479-5), CNPQQ (número de financiamento: 308162 / 2014-5) e Coordenação de Aperfeiçoamento de Pessoal de Nível Superior Código Financeiro 001 da CAPES. Os autores negam quaisquer conflitos de interesse.

\section{Referências}

Araújo-Reis, C., Araújo, S. S., Baratto-Filho, F., Reis, L. C., \& Fidel, S. R. (2009). Comparação da infiltração apical entre os cimentos obturadores AH Plus, Sealapex, Sealer 26 e Endofill por meio da diafanização. Rsbo. 1(6):21-28.

Artopoulou, I. I., O'Keefe, K. L., \& Powers, J. M. (2006). Effect of core diameter and surface treatment on the retention of resin composite cores to prefabricated endodontic posts. J Prosthodon. 15(3):172-9.

Balbosh, A., Kern, M. Effect of surface treatment on retention of glass-fiber endodontic posts. J Prosthet Dent (2006). 95(3):218-23.

Benassi, M., Freire, R. M., Macedo, M. C., \& Cardoso, R. J. A. (2008). Avaliação da superfície dentinária com o microscópio clínico após remoção de retentor intra-radicular utilizando o ultra-som. RGO. 56(3):267-273.

Braga, N. M. A., Alfredo, E., Vansan, L. P., Fonseca, T. S., Ferraz, J. A. B., \& Sousa-Neto, M. D. (2005). Efficacy of ultrasound in removal of intraradicular posts using diferente techniques. J Oral Sci; 47(3):117-21.

Coelho, E. T., \& Sousa, T. L. P. Pinos de fibra de vidro: Protocolos de desobstrução no retratamento endodôntico.40 f. TCC (Graduação) - Curso de Odontologia, Universidade Potiguar, Natal, 2012.

Fracassi, L. D., Ferraz, E. G., Albergaria, S. J., \& Sarmento, V. A. (2010). Comparação radiográfica do preenchimento do canal radicular de dentes obturados por diferentes técnicas endodônticas. Rev Gaúcha Odontol., 58(2): 173-179.

Garrido, A. D. B. Avaliação de diferentes protocolos de aplicação de ultra-som para remoção de retentores intraradiculares fundidos fixados com cimento de fosfato de zinco. 2007. 107 f. Tese (Doutorado) - Curso de Odontologia, Universidade de Ribeirão Preto, Ribeirão Preto, 2007.

Gesi, A., Magnolfi, S., Goracci, C., \& Ferrari, M. (2003). Comparison of two techniques for removing fiber posts. J Endod. 29(9): 580-2.

Gonçalves, B. E. M., Silva, S. J. A., Da-Araújo, R. P. C. (2012). Avaliação da eficácia obturadora do Coltosol® e do IRM® no selamento provisório de dentes sob intervenção endodôntica. $R$. Ci. med. biol. 11(2): 154-158.

Goodacre, C. J. (2010). Carbon fiber posts may have fewer failures than metal posts. J Evid Base Dent Pract; 10(3): $32-4$.

Kalkan, M., Usumez, A., Ozturk, N. A., Belli, S., Eskitascioglu, G. (2006). Bond strength between root dentin and three glass-fiber post systems. J Prosthet Dent. 96(1):41-6.

Kim, J. J., Alapati, S., Knoernschild, K. L., Jeong, Y. H., Kim, D. G., \& Lee, D. J. (2016). Micro-computed tomography of tooth volume changes following post removal. J Prosthodon. 1-7.

Lindemann, M., Yaman, P., Dennison, J. B., \& Herrero, A. A. (2005). Comparison of the efficiency and effectiveness of various techniques for removal of fiber posts. J. Endod. 31(7):520-522.

Lopes, H. P., \& Siqueira Junior, J. F. (2010). Endodontia: Biologia e técnica. (3a ed.), Editora Guanabara Koogan.

Muniz, L. (2005). Novo conceito para retenção intra-radicular: Preparo endodôntico para pinos de fibra. R Dental Press Estét. $2(1): 70-8$.

Oliveira, A. C. M., \& Duque, C. (2012). Métodos de avaliação da resistência à infiltração em obturações endodônticas. Revista Brasileira de Odontologia. 69(1): 34-38. 
Research, Society and Development, v. 10, n. 2, e3481029536, 2021

(CC BY 4.0) | ISSN 2525-3409 | DOI: http://dx.doi.org/10.33448/rsd-v10i2.9536

Pereira, A. D., Shitisuka, D. M., Parreira, F. J., \& Shitsuka, A. R. Metodologia de pesquisa cientifica. UFSM. https://www.ufsm.br/orgaossuplementares/nte/wpcontent/uploads/sites/358/2019/02/Metodologia-da-Pesquisa-Cientifica_final.pdf.

Rijk, W. G. Removal of fiber posts from endodontically treated teeth. American J Dent. 13(2):19-21.

Rocha, I. A. R. Avaliação da eficácia e eficiência na remoção de pino de fibra de vidro. 20p. Monografia - Faculdade de Odontologia UFBA. Salvador/BA

Rossi, M. Técnicas para remoção de pinos intra-radiculares.2008. 33p. Dissertação (Especialização) - Faculdade Ingá, UNINGÁ, Passo Fundo/RS.

Silva, E. J., Neves, A. A., De-Deus, G., Accorsi-Mendonça, T., Moraes, A. P., Valentim, R. M., \& Moreira, E. J. (2015). Cytotoxicity and gelatinolytic activity of a new silicon-based endodontic sealer. J Appl Biomater Funct Mater. 13(4):376-80.

Soares, J. A., Brito-Júnior, M., Fonseca, D. R., Melo, A. F., Santos, S. M. C., Sotomayor, N. D. C. S., et al. (2009). Influence of luting agents on time required for cast post removal by ultrasound: an in vitro study. J Appl Oral Sci. 17(3):145-9 